\title{
Handover Mechanisms in ATM-Based Mobile Systems
}

\author{
G. Karagiannis*, M.C. de Lignie**, J. de Bie** and I.G.M.M. Niemegeers* \\ (*) Centre for Telematics and Information Technology \\ University of Twente \\ P.O. Box 217, 7500 AE Enschede, The Netherlands \\ (**) KPN Research \\ P.O. Box 421, 2260 AK Leidschendam, The Netherlands
}

\begin{abstract}
This paper presents two handover mechanisms that can be used in the access part of an ATM-based mobile system. The first handover mechanism, which is called "handover synchronised switching" is relatively simple and does not use any ATM multicasting or resynchronisation in the network. It assumes that there is sufficient time available such that all data and history information of the old path can be transferred to the Mobile Terminal (MT) before the actual handover to the new path takes place. It is possible that the time between a handover decision and the actual handover is too short to end the transmission on the old path gracefully (e.g., ending the interleaving matrix, ending transcoder functions, emptying intermediate buffers). A possible solution to this problem is given by the second handover mechanism, where multicast connections to all possible target Radio Systems (RAS) are used in the core network. This mechanism is called "handover with multicast support".
\end{abstract}

\section{INTRODUCTION}

The use of mobile communications has increased rapidly during the 80 's and 90 's. This growth started in 1980 , when the first generation analogue cellular phones were introduced in the business sector. Second generation digital systems have recently been introduced. It is expected that these second generation systems will reach the limits of their capacity early in the next century. It is predicted that by the year 2000 the market in Europe will have grown to over 100 million users. By that time there will be a need for a new generation of mobile telecommunication systems. The MONET project (see [MON113], [Bui95]), sponsored by the EEC as part of the RACE 2 programme, aimed to develop a third generation system called Universal Mobile Telecommunication System (UMTS). UMTS is intended to be a system capable of providing a diversity of services (requiring up to $2 \mathrm{Mbit} / \mathrm{s}$ ). In addition to multiparty and multi-media services, it should integrate services now being offered by cordless, cellular, (dedicated) paging and private mobile radio systems. It should be operational in different environments such as domestic, business, vehicular, and multi-operator public environments. In the MONET project, the target in the development of the UMTS is that B-ISDN (ATM based) will be used as the backbone network and that the IN (Intelligent Network) will be applied to implement the UMTS mobility features. The basic UMTS architecture (Figure 1) [MON113] consists of an RAS (Radio Access Network) composed of the B'TS (Base Transceiver Station) a CSS (Cell Site Switch), and a M-SCP (Mobility and Service Control Point) and a backbone network composed of the LE, TX and M-SCP. The Mobile Terminal (MT) and the FT represent the mobile and fixed end users. The CSS takes care of basic call control and connection to the fixed core network. The radio access for MT's is provided via BTS's. The MSCPs comprise the functionality for mobility and service control, whereas the M-SDPs model the mobility data storage. Signalling System 7 (SS7) is used to control the flow of information between the interactive network functional entities to establish, maintain and release an IN service request, initiated by a mobile or fixed user.

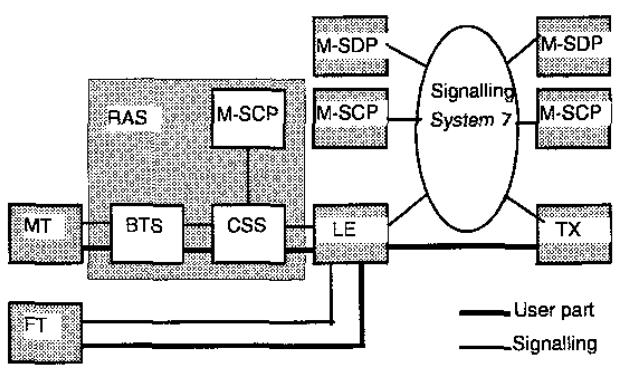

Figure 1. Overview of the basic UMTS architecture

\section{MOBILITY AND HANDOVER}

An MT can gain access to the UMTS core network through an RAS. The MT is able to roam anywhere in the UMTS access system. The handover can be described as the algorithm that automatically transfers a transaction in progress, i.e., the MT connection, to each new BTS when the MT by roaming changes radio channels and/or connections with the old BTS. The handover issue for second generation mobile systems has been studied and 
presented in several papers such as [Huss94], [CoWi96], [Brem95], [SeEv95], [Whin95]. In third generation systems, the handover procedure becomes more complex due to the fact that high speed advanced multiparty and multimedia services will have to be supported by these systems. Therefore more efficient handover algorithms will have to be developed. In [VeKa95] a handover algorithm, called "Homing Algorithm", is provided for an wireless ATM LAN (Local Area Networks) that is able to support multimedia PCS applications. This algorithm is able to deliver the ATM cells to the MT's in their proper FIFO sequence. A different handover algorithm is presented in [CoPa96]. This algorithm is a distributed handover protocol, and proves that it avoids loss of packets and preserves order of transmission as long as the mobile stays in one region. In [Toh97] the problems associated with handovers of multicast ATM connections are highlighted and a methodology is revealed to support both sender and receiver handovers of multicast connections. In this paper two new handover mechanisms that affect both user (transport) and signalling (control) parts of the UMTS system are presented. The first handover algorithm is relatively easy and it does not use any difficult ATM multicasting and re-synchronisation in the network. It assumes that there is sufficient time available such that all data and history information of the old path can be transferred to the Mobile Terminal (MT) before the actual handover to the new path takes place. This first algorithm is called "handover synchronised switching". It is possible that the time between a handover decision and the actual handover is too short to end the transmission on the old path gracefully (e.g., ending the interleaving matrix, ending transcoder functions, emptying intermediate buffers). A possible solution for this problem is given by the second handover algorithm where multicast connections are used in the core network. This algorithm is called "handover with multicast support".

The handover mechanisms are presented by using both the transport and control descriptions.

\section{HANDOVER SYNCHRONISED SWITCHING}

The handover (HO) synchronised switching mechanism features a make-before-break scenario, where enough time is available to the network for handing over connections between two Radio Access Systems. The initial assumption is that during a sufficiently long interval, both the old RAS and the new RAS can be reached by the MT. This scenario is feasible if the filling of the buffers at the time of $\mathrm{HO}$ is not heavy.

\section{A. Transport description}

Figure 2 depicts the consecutive steps for performing $\mathrm{HO}$ at transport level, according to the scenario described above. Only the involvement of the ATM backbone network is discussed and the focus is on the execution phase (see [MON113]). A short description of the four steps follows:

Phase 1.: The MT communicates both upstream and downstream with the old RAS. The old RAS communicates in both directions with the Local Exchange (LE). The MT indicates to the network that it is about to roam into a (specific) new RAS. Handover is initiated by activating the appropriate signalling procedures to set up the new path in advance, before the actual HO switching occurs.
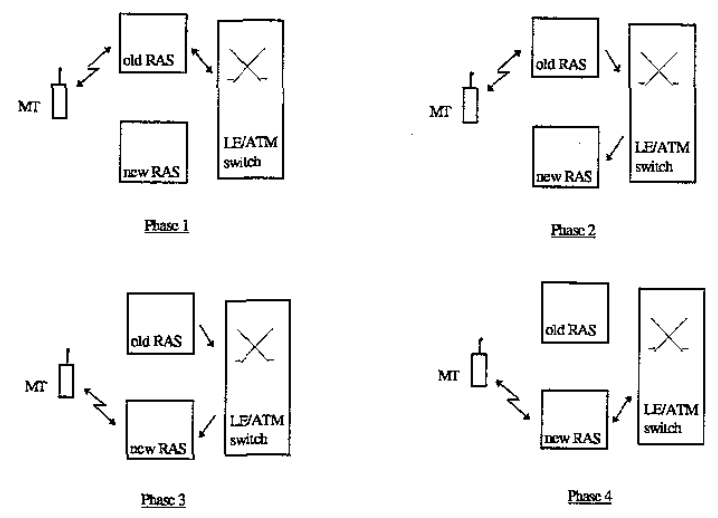

Figure 2. The synchronised switching ("sufficient time") HO transport description

Phase 2: The new path is setup, a buffer for upstream data is placed in the LE and a buffer for downstream data in the new RAS. The MT communicates up and down with the old RAS. The LE switches the downstream flow to the new path, indicating this to the old RAS. The new RAS starts buffering the downstream data that it receives from the LE. The old RAS communicates in the upward direction with the LE, while emptying its downstream buffers to the MT.

Phase 3: The downstream buffers of the old RAS are empty. Upon this indication, the MT switches over to the new RAS and starts receiving its buffered data. The LE keeps sending downstream data to the new RAS. The MT starts sending its upstream data to the new RAS and LE. The LE buffers the upstream data from the MT. The old RAS empties its upstream buffers to the LE.

Phase 4: The upstream buffers of the old RAS are empty. This is indicated to the LE. The old RAS is no longer involved in the connection. The LE redirects the upstream connection to the new path and starts sending its buffered upstream data into the network. The LE initiates the appropriate signalling procedures to release the old path. The MT communicates both up and down with the new RAS. The new RAS communicates both up and down with the LE. 
It is assumed that all paths involved in HO use ATM Virtual Channel Connections (VCCs). Although VCCs are bi-directional, different approaches for upstream and downstream $\mathrm{HO}$ are possible, i.e., the switching instances for upstream and downstream traffic can be separated in time.

\section{B. Control description}

The main task in defining the control of the synchronised switching handover scenario is to translate the phases of the transport plane as described above into states of the signalling protocols at all the interfaces involved. This includes the use of special ATM delimiter cells in the transport plane to control the time critical steps of the handover procedure.

This section uses the network configuration of Figure 2 , which is valid for handovers that involve different RASs. The actual control of the synchronised switching handover procedure is described in the following sequence of steps and in Figure 3. The circled numbers in Figure 3 identify the step in which each message is sent or received. The ATM delimiter cells are also drawn as signalling messages (dashed arrows), because they cause a state change in the signalling protocol state machines. During this control procedure the following steps are accomplished (see also Section III-A):

1. The MT starts the HO initiation procedure (see [MON113]) when it is moving from the old RAS to the new RAS. This is Phase 1 of the transport description.

2. The $\mathrm{HO}$ execution procedure is started when the $\mathrm{LE}$ receives an $\mathrm{HO}$ execution request from the MSCP.

3. By using the signalling plane the LE connects a new path through the LE, the new RAS and the MT. From the content of the setup request [MON99] it is clear to the new RAS and the MT that it is not an ordinary setup procedure but one related to handover of an existing connection. This is Phase 2 of the transport description. During this step the following actions are accomplished:

a. the transport plane of the new RAS is activated such that a buffer is allocated for transport cells received from the $\mathrm{LE}$ in the downstream direction. The new RAS will not send transport cells downstream to the MT until it receives further instructions.

b. the transport plane of the MT is prepared to receive a delimiter cell from the old RAS and switch over to the new RAS. Meanwhile, the MT keeps sending and receiving cells from the old RAS.

c. after the new path has been established, the transport plane in the LE is activated such that firstly it will mark the last cell sent downstream to the old RAS. Secondly, it will send subsequent transport cells downstream to the new RAS. Thirdly, it will allocate a buffer to queue any cells received upstream from the new RAS (this buffer may remain empty, depending on the time lag between the old and new paths).

When the old RAS receives the last marked cell, it knows that it must prepare to end the downstream transmission on the radio path. The old RAS still transfers the last marked cell to the MT or gives some other indication via the radio protocols that the MT can switch to the new RAS. It is assumed that the old RAS is able to stop transmission after the last ATM cell without any cell loss, e.g., by stuffing any interleaving frames with zeros, because the LE has no means of knowing the right time to switch the downstream path to the new RAS. This assumption can be considered to be a requirement of the synchronous switching scenario to the radio interface.

4. When the MT receives a delimiter cell in the transport plane, indicating that the old RAS has emptied its downstream buffer, it will subsequently send cells upstream to the new RAS. This is Phase 3 of the transport description. The first cell sent is a delimiter cell. Now the MT also expects to receive cells from the new RAS.

5. When the new RAS receives through the transport plane the marked cell that was sent upstream by the MT, it subsequently reactivates the transport plane and sends the queued cells from the downstream buffer downstream to the MT. The new RAS transfers the upstream cells received from the MT to the LE.

6. When the old RAS has received the last upstream cell from the MT it adds a delimiter cell and starts emptying its upstream buffer. The old RAS has to conclude that the MT has sent the last cell when the radio link with the MT has disappeared (i.e., when the MT has switched to the new RAS). This is Phase 3 of the transport description. When the last cell queued in the upstream buffer of the old RAS is received by the LE, the LE also switches the upstream path from the old to the new RAS and starts emptying its upstream buffer associated with the upstream path from the new RAS, by sending the cells upstream into the network.

Subsequently, the LE simultaneously starts the "release of the old path" procedure through the old path and the "handover finish" procedure through the new path. The "handover finish" procedure acknowledges to the new RAS and the MT that the handover procedure was completed successfully and that all allocated resources (e.g., buffers) related to the handover procedure can be released. This is Phase 4 of the transport description.

7. The handover execution procedure is completed. Some further remarks:

- The use of delimiter cells between the MT and the RAS should not be taken too literally. The radio protocols may have other means to implement the time critical control, e.g., some indication in the interleaving headers. 
- The scenario above only covers successful handover. Procedures have to be added to account for lost delimiter cells. Acknowledgement of receipt of a delimiter cell could be realised via a control plane signalling message. Then a timer could be set to retransmit the delimiter cell if no acknowledgement is received.

- In the situation where radio protocols initiate the new upstream path (see step 4) from the MT to the new RAS then the MT will not have to send a begin-delimiter upstream to the new RAS.

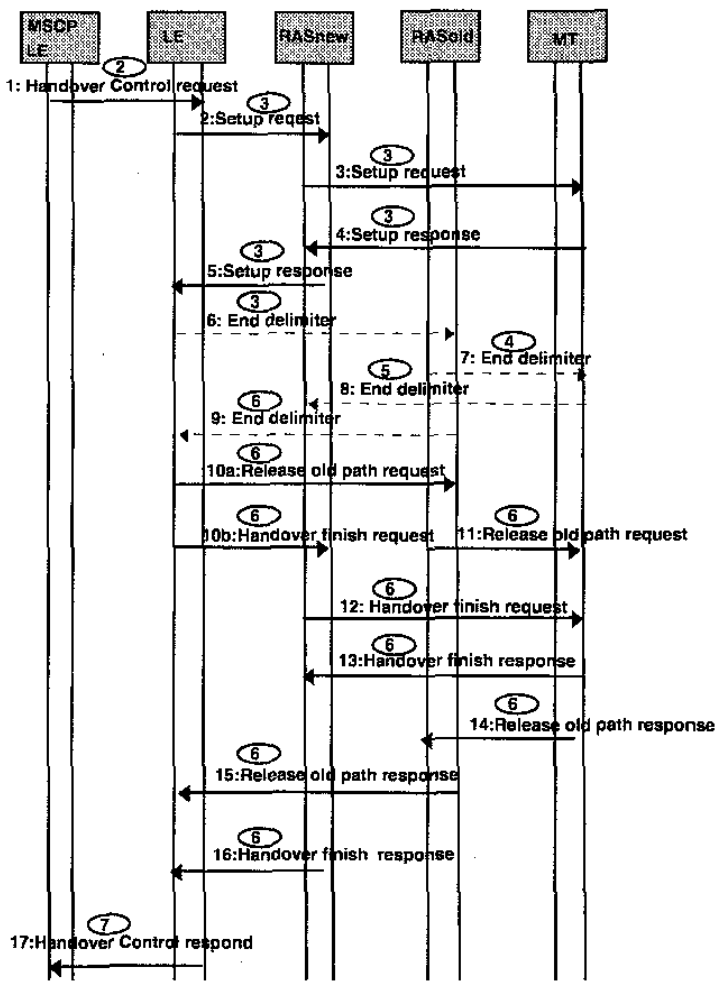

Figure 3. The HO execution control message flows

\section{HANDOVER WTTH MULTICAST SUPPORT}

When the time between a handover decision and the actual handover is too short to end the transmission on the old path gracefully (e.g., ending the interleaving matrix, ending transcoder functions, emptying intermediate buffers) a different handover mechanism has to be used. This mechanism is called handover with multicast support'.

\section{A. Transport description}

This mechanism uses multicast connections in the core network. By using multicast connections to all possible new target RAS systems, the history can already be built up on the possible target paths. At the time the handover needs to take place the history on the old path can be ignored, and the history on the new path can be used to resume transmission.

Multicast could also be used for the upstream direction. However using multicast in the upstream direction, complicates the design of the LE and the RAS considerably without giving many advantages. If both the old and the new RASs are reachable for some time one can use this time to gracefully end any interleaving and/ or transcoding on the old path before going to the new path.

\section{HO with Multicast Support Phases}

Figure 4 depicts the consecutive phases for performing HO at transport level for the downstream direction and Figure 5 depicts the consecutive phases for the upstream direction. Note that each rectangle that encompasses the following terms DA, DB, DC and DD represents a combination of input and output buffers used in the downstream direction while each rectangle that encompasses the terms UA, UB, UC and UD represents a combination of input and output buffers used in the upstream direction. The marked buffers (coloured grey) represent the ones that contain cells which are being processed during that phase.
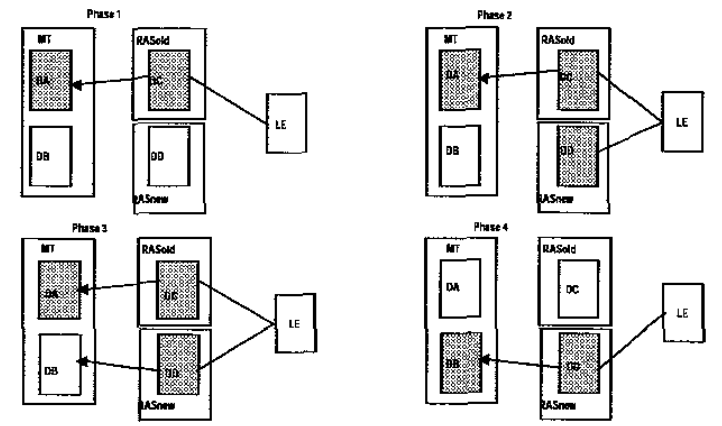

Figure 4. HO with multicast support for the downstream direction.

\section{HO Downstream with Multicast Support}

The HO mechanism for the downstream direction is depicted in Figure 4. Note that this scenario includes a phase 3 where the MT receives cells from both the new RAS and the old RAS. This phase requires additional resources on the radio path and should be kept as short as possible. It would even be possible to use a synchronised switching method on the access interface such that this phase is not necessary (direct transition from phase 2 to phase 4).

Phase 1

The LE communicates downstream with the old RAS (DC) and the old RAS communicates with the MT (DA). 


\section{Phase 2}

- The LE adds a path to the connection. The LE will now start replicating the information and send it to both the old RAS (DC) and the new RAS (DD). The new RAS can build up the required interleaving and/or transcoding history.

- Because the data going over the old and new paths must eventually be synchronised again in the MT, synchronisation information needs to be included in the data stream. If we do not have synchronisation information already in the information stream coming from the originating $\mathrm{MT}$, we need to add this synchronisation information before or at the splitting point. One possible method is to insert specially marked cells (e.g., Operation And Management (OAM) cells with some sort of sequence number) in the data stream. These marked cells will be sent in both directions. Both the new and the old RAS should include this synchronisation information in the data they send to the MT.

- The MT processes the synchronisation points and it starts counting information units (messages) between synchronisation points.

- Information in the new RAS that become outdated before it can be sent to the MT should be discarded. Information can become outdated either because of a maximum time in the buffer or because of an acknowledgement received from the MT.

- If the simultaneous transmission over the old and new radio paths is possible, the new RAS can start transmitting data over the new path (transition to phase 3 ). If simultaneous transmission is not possible a direct transition from phase 2 to phase 4 is required.
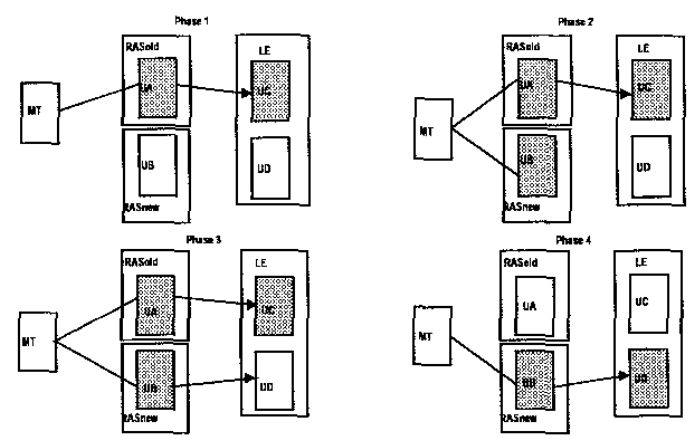

Figure 5. HO with multicast support for the upstream direction.

\section{Phase 3}

- If the quality of the new radio path is good enough, the new RAS can start sending information to the MT.

- In this situation the MT receives downstream two streams of cells, one from the old RAS (DC) that it will store into the DA buffer and one from the new RAS
(DD) that it will store into the DB buffer. The MT keeps processing the cells from the old RAS (buffered in DA).

- The MT starts sending an acknowledgement to the new RAS to indicate the point in the datastream that was already received from the old path. The new RAS can use this acknowledgement to discard any old information (that was already sent).

- The streams of cells received are not synchronised. The new path can either be shorter or longer than the old path. So the new cell stream may be either in front of or behind the old cell stream.

\section{Phase 4}

- The MT cannot receive any more cells from the old RAS (transmission related). At a suitable moment in time the MT will switch from the old buffer to the new buffer. Before this processing begins, the two buffers need to be synchronised. All the information that was already processed from the old buffer needs to be removed from the new queue. Outdated data can be recognised by looking at the synchronisation points and counting the information that was sent after the last synchronisation point.

- If the new path is running somewhat behind the old path, the entire new buffer must be removed, moreover an additional amount of data must still be received from the new RAS.

- If the information on the new path is running far behind the old path, a mechanism needs to be used to remove outdated information inside the new RAS. In this situation the MT can indicate to the new RAS the exact point in the information that was already received from the old path. The new RAS can use this information to remove all outdated information.

- At the end of phase 4 the LE sends cells only to the new RAS (DD) and the new RAS (DD) sends cells only to the MT (DB).

\section{HO Upstream with Multicast}

The HO mechanism for the upstream direction is shown in Figure 5.

\section{Phase 1}

- The MT communicates upstream with the old RAS (UA), the old RAS (UA) communicates upstream with the LE (UC).

\section{Phase 2}

- The MT sends cells to both the old RAS (UA) and to the new RAS (UB). The MT must insert synchronisation information into the information stream (e.g., specially marked cells are added in the information stream), so the new RAS can build up the required interleaving and/or transcoding history.

- If the LE has the possibility of buffering the cells coming from the new path, the new RAS can already start transmitting cells to the LE. In this situation the 
system can move into phase 3 . If the LE does not have this possibility a direct transition to phase 4 is required.

\section{Phase 3}

- The LE receives upstream two streams of cells, one from the old RAS (UA) that will be stored into the UC buffer and one from the new RAS (UB) that will be stored into the UD buffer. The received cells are not yet synchronised when they arrive at the LE. In order to enable eventual synchronisation the LE may need to count the cells that have been sent after the last synchronisation point.

\section{Phase 4}

- In both phases 2 and 3 the MT sends cells to the new RAS (UA) and the old RAS (UB). At a certain moment in time the MT will decide that a handover is required. At this time a special indication will be sent upstream over the old path to the LE.

- If the LE receives the handover indication from the old RAS, it must switch over to the new path. If the previous handover phase was phase 3 , it means that the LE will have to switch internally to another buffer. If the previous handover phase was phase 2 , it means that the new RAS should be told to start sending data over the new path to the $\mathrm{LE}$.

- Before starting, all outdated information (i.e., information that has already been sent) must be removed. Outdated data can be recognised by looking at the synchronisation points and counting the information that has been sent after the last synchronisation point.

- At the end of phase 4 the MT sends cells only to the new RAS (UB) and only the new RAS (UB) sends cells to the LE (UD).

\section{B. Control description}

This section uses the network configurations depicted in Figures 4 and 5. The sequence of steps involved in the actual control of the handover with multicast procedure is described below and is depicted in Figure 6. The circled numbers in Figure 6 identify the control action step in which each message is sent or received. The ATM delimiter cells are also drawn as signalling messages (dashed arrows), because they cause a state change in the signalling protocol state machines. During this control procedure the following steps are accomplished (see also Section IV-A):

1. The MT starts the HO initiation when it is moving from the old RAS to the new RAS.

2. The HO execution procedure is started when the $\mathrm{LE}$ receives a $\mathrm{HO}$ execution request from the MSCP.

3. By using the signalling plane the $L E$ adds a new path through the LE, new RAS and the MT. This is achieved by using an add party procedure that is similar to the one described in [B-ISDN]. Note that this procedure uses additional mobility information required in UMTS [MON113]. From the content of the add party request it is clear to the new RAS and the MT that it is not an ordinary B-ISDN add party procedure but one related to handover of an existing connection. During this step the following actions are accomplished:

a. The transport plane of the new RAS is activated such that transport cells received from the $\mathrm{LE}$ will be queued into the downstream buffer.

b. The transport planes in the MT and LE are prepared to first receive a delimiter cell from the old RAS and to reset the counters that are registering the number of processed cells, second the MT and LE will receive on the transport part subsequent delimiter cells from old and new RAS used to synchronise the two downstream buffers (DA and DB) located in the MT and the two upstream buffers (UC and UD) located in the LE; third the MT will mark the last cell that will be sent upstream, while the LE will mark the last transport cell that will be sent downstream to the old RAS; fourth the MT will send transport replicated cells upstream and the LE will send replicated cells downstream to the new and old RAS. During this step delimiter cells will be sent from the MT upstream, and from the LE downstream on both paths (old and new) to synchronise the UC, UD, and DA, DB buffers. Fifth, the LE will queue any cells received upstream and the MT will queue any cells received downstream from the new and old RAS.

4. When the MT starts processing a delimiter cell that indicates that the old RAS has emptied the cells that were processed before the HO initiation procedure started, it will reset a counter that will be used to register all the processed cells by the MT. Subsequently the MT will receive the marked cell used to synchronise the two buffers DA and DB.

5. Similarly to the control action 4 , the LE receives through the transport plane the marked transport cell that was sent upstream by the MT, it will reset a counter that will be used to register all the processed cells by the LE. Subsequently the LE will receive the marked cell used to synchronise the two buffers UC and UD.

6. When the MT is not able to receive downstream transport cells because of transmission problems, it will start processing cells that were sent by the new RAS (buffer DB). Before starting with this processing, the following procedure must be used. The two buffers DA and DB need to be synchronised. All the information that was already processed from the old buffer DA needs to be removed from the new queue DB. Outdated data can be recognised by looking at the synchronisation points (delimiter cells) and counting the information that was sent after the last synchronisation point. If the new path is running somewhat behind the old path, the entire new buffer (DB) must be removed. If the information on the new path is running far behind the old path, a mechanism must also be used for removing outdated information inside the new RAS. In this situation the MT can indicate 
to the new RAS the exact point that was already received from the old path. The new RAS then activates the transport part such that all outdated information will be removed from the buffer DD. Note that this procedure can be accomplished during the "handover finish" procedure (see step 7).

7. This control action step is similar to step 6, with the difference that the actions described in step 6 are performed on the LE and on the buffers UC and UD. If the LE is not able to receive upstream transport cells because of transmission problems, through the transport part, it will start processing cells that were send by the new RAS (buffer UD). Before starting with this processing, the following procedure must be used. The two buffers UC and UD need to be synchronised. All the information that was already processed from the old buffer (UC) needs to be removed from the new queue (UD). Outdated data can be recognised by looking at the synchronisation points (delimiter cells) and counting the information that was sent after the last synchronisation point. If the new path is running somewhat behind on the old path, the complete new buffer (UD) must be removed. If the information on the new path is running far behind on the old path, a mechanism also needs to be used for removing outdated information inside the new RAS. In this situation the LE can indicate to the new RAS the exact point that was already received from the old path. The new RAS then activates the transport part such that all outdated information will be removed from the buffer UB. Note that this procedure can be accomplished simultaneously with the normal procedure of sending and receiving ATM cells through the transport part. Subsequently, the LE will start simultaneously the B-ISDN "detach party" [B-ISDN] procedure through the old path and the "handover finish" procedure through the new path. The "handover finish" procedure acknowledges to the new RAS and the MT that the handover procedure was completed successfully and that all allocated resources related to the handover procedure can be released. Note that the "detach party" and the "handover finish" procedures can also be started by the MT. During the "handover finish" procedure the new RAS can be informed by the MT or the LE of the exact point in the information already received from the old path. The new RAS can then use this information to remove all outdated information.

8. The HO execution procedure is completed.

The additional remarks related to the control of the $\mathrm{HO}$ mechanism given in the previous section can also be applied to this section.

The HO synchronised switching mechanism can e.g., be used in highway areas where no obstacles influence the handover procedure. However in metropolitan centre areas, where a significant number of obstacles exist, e.g., high building corners, the $\mathrm{HO}$ with multicast support mechanism is a better candidate.

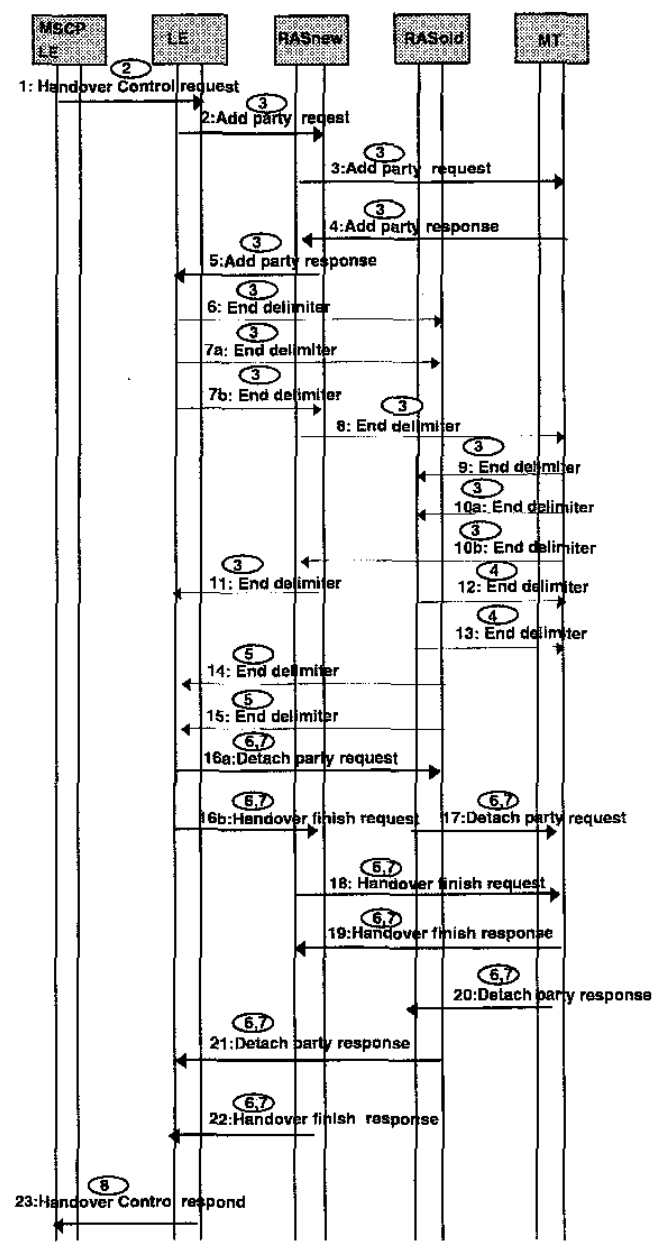

Figure 6. The HO execution control message flows.

\section{CONCLUSIONS}

This paper presents two $\mathrm{HO}$ mechanisms. The first mechanism is the HO synchronised switching mechanism and the second is the $\mathrm{HO}$ with multicast support mechanism. The first handover mechanism is relatively simple and does not use any ATM multicasting or resyncronisation in the network. The scenario assumes that there is sufficient time available for all the data and history information of the old path to be transferred to the MT before the actual handover to the new path takes place. This means that time is available to empty the buffers on the old path and to stop the interleaving and transcoding process on the old path. If the time between a handover decision and the actual handover is too short to end the transmission on the old path gracefully (e.g., ending the interleaving matrix, ending transcoder functions, emplying intermediate buffers), then a possible alternative is given by the second HO mechanism, where in the downstream direction multicast connections are 
used in the core network. By using multicast connections to all possible new target RAS systems, the history can already be built up on the possible target paths. At the time the handover needs to take place, the history on the old path can be ignored and the history at the new path can be used to resume transmission. The operation of the HO mechanisms require the use of end delimiters. When information is switched between old and new paths (both up- and down- stream) the last information on the old path is marked with an end delimiter. The destination will receive information from the old path until this end delimiter and then switch to receiving information from the new path. The end delimiter might be implemented in the ATM header or as an Operations and Maintenance ATM cell.

Acknowledgements. This paper is based on work conducted by the RACE II project MONET (Mobile Networks) in which the authors participated. The views expressed in this paper are those of the authors and not necessarily those of the project as a whole. The members of the MONET project, in particular Toon Norp and Marion Rafalli are acknowledged for their help and discussion.

The authors would also like to express their gratitude to Philip Chimento, Bert Jan van Beijnum, Val Jones and Victor Nicola for their discussion on the subject, and to the European Commission for sponsoring the RACE programme.

\section{REFERENCES}

[B-ISDN] ITU/SG11, "Broadband capability set 2 signalling requirements", Question 10/11, December 1993.

[Brem95] Bremer R., "Inter-PLMN handover-an approach for a functional requirement description", Proc. of Fourth IEEE International Conference on Universal Personal Communications, p. 442-6, 1995.

[Bui95] Evert Buitenwerf, "RACE 2066 MONET: UMTS fixed network specifications", Mobile Summit, Cascais Portugal, November 1995.

[CoPa96] R. Cohen, B. V. Patel, A. Segall, "Handover in a micro-cell packet switched mobile network", Journal of Wireless Networks Iss. 2, pp. 13- 25, 1996.

[CoWi96] Constantini, M., Wilkinson, T.A., Pupolin, S, "Handover issue in DECT systems", Journal Electronics Letters, Vol. 32, Iss. 3, pp. 174-175., 1996.

[Huss94] Hussmann, $\mathrm{H}$, "Performance analysis of handover and channel management schemes for indoor scenarios based on ray tracing techniques", Proc. of 44th Vehicular Technology Conference, 1994.
[MON113] R2066/BT/PM2/DS/P/113/b1, "UMTS System Structure Document", December 1995.

[MON99] R2066/PTT NL/MF/DS/P/99/b2, "Baseline document on logical and functional models", December 1995.

[SeEv95] Senarath G.N., Everitt D., "Performance of handover priority and queueing systems under different handover request strategies", 1995 IEEE 45th Vehicular Technology Conference, Vol.2, p. 897-901.

[Toh97] C-K. Toh, " A unifying methodology for handovers of heterogeneous connections in wireless ATM networks", Computer Communication Review, Vol. 27 (1) pp. 12 - 30, 1997.

[VeKa95] M. Veeraraghavan, M. Karol and K.Y. Eng, "Mobility management in a wireless ATM LAN", GLOBECOM'95, Vol. 1, pp.316 - 321, 1995.

[Whin95] Whinnett N.W., "Handoff between disimmilar systems: General Approaches and air interface issues for TDMA systems", Proc. of 45th IEEE Vehicular Technology Conference, Vol.2, p. 953-957, 1995. 